\title{
Parenteral nutrition including an omega-3 fatty- acid-containing lipid emulsion for intensive care patients in China: a pharmacoeconomic analysis
}

This article was published in the following Dove Press journal:

ClinicoEconomics and Outcomes Research

5 September 2017

Number of times this article has been viewed

\author{
Yufei Feng' \\ Chao $\mathrm{Li}^{1}$ \\ Tian Zhang' \\ Lorenzo Pradelli
}

'Department of Pharmacy, Beijing

Hospital, National Center of

Gerontology, Beijing, People's

Republic of China; ${ }^{2}$ AdRes Health

Economics and Outcomes Research,

Piazza Carlo Emanuele II, Torino, Italy
Background/objectives: Parenteral nutrition (PN) incorporating omega-3 fatty-acid-enriched lipid emulsions has been shown to be cost effective in Western populations. A pharmacoeconomic evaluation was performed within the Chinese intensive care unit (ICU) setting. This assessed whether the additional acquisition cost of PN with omega-3 fatty-acid-enriched lipid emulsion (SMOFlipid) vs standard PN was offset by improved clinical outcomes that can reduce subsequent costs.

Materials and methods: A pharmacoeconomic discrete event simulation model was developed, based on an update to efficacy data from a previous international meta-analysis, with China-specific clinical and economic input parameters. Sensitivity analyses were undertaken to assess the effects of uncertainty around input parameters.

Results: The model predicted that PN with an omega-3 fatty-acid-enriched lipid emulsion was more effective and less costly than PN with standard lipid emulsions for Chinese ICU patients, as follows: reduced length of overall hospital length of stay (19.48 vs 21.35 days, respectively), reduced length of ICU stay (5.03 vs 6.18 days, respectively), and prevention of $35.6 \%$ of nosocomial infections leading to a lower total cost per patient ( $¥ 47189$ [US \$6937] vs $¥ 54783$ [US \$8053], respectively). Additional treatment costs were offset by savings in overall hospital and ICU stay cost, and antibiotic cost, resulting in a mean cost saving of $¥ 7594$ (US \$1116) per patient. Sensitivity analyses confirmed the robustness of these findings.

Conclusions: PN enriched with an omega-3 fatty-acid-containing lipid emulsion vs standard PN may be effective in reducing length of hospital and ICU stay and infectious complications in Chinese ICU patients, and also decreases overall treatment costs. This results in a favorable cost-effectiveness ratio. Thus, PN enriched with an omega-3 fatty-acid-containing lipid emulsion can be seen as a win-win situation for patients, hospital administration, and health insurance companies.

Keywords: parenteral nutrition, omega-3, lipid emulsion, cost effectiveness, intensive care, China

\section{Introduction}

There is considerable evidence that disease-related malnutrition increases mortality rates, prolongs the length of hospital stay, and increases overall treatment costs. ${ }^{1-3}$ For example, malnutrition is an independent risk factor for nosocomial infection, which can be up to five times more likely in malnourished than well-nourished hospital patients. ${ }^{4,5}$ Moreover, disease-related malnutrition of hospital patients is an extensive problem, having a worldwide prevalence of about $20 \%-50 \%,{ }^{1,6}$ which is also reflected in the People's Republic of China where there is a prevalence of about $18 \%-48 \%{ }^{7-10}$ The underlying
Correspondence: Lorenzo Pradelli AdRes Health Economics and Outcomes Research, Via Vittorio Alfieri, 17-10123, Turin, Italy

Tel +39 0II 765 I239

Email l.pradelli@adreshe.com 
reason for disease-related malnutrition within hospitals and particularly in the intensive care unit (ICU) is that a negative energy balance accumulates during the course of some serious diseases and after major surgery, and these energy deficits are associated with worsened clinical outcomes. ${ }^{11,12}$

Nutritional interventions for hospital patients are known to reduce the incidence of nosocomial infections, ${ }^{12,13}$ length of hospital stay, ${ }^{14,15}$ and medical costs. ${ }^{16}$ Parenteral nutrition is a common nutritional intervention, and is indicated when enteral or oral nutrition is impossible, insufficient, or contraindicated. ${ }^{17}$ The composition of parenteral nutrition is particularly important, and lipids are an essential component as a source of energy and essential fatty acids. ${ }^{17}$

Omega-3 fatty-acid-enriched lipid emulsions are commonly used as part of parenteral nutrition, and are recommended in nutritional guidelines for parenteral nutrition in ICU and surgical patients. ${ }^{17,18}$ This is because omega-3 fatty-acid-enriched lipid emulsions may have beneficial anti-inflammatory and immunomodulatory effects. ${ }^{19-21}$ While the pharmacoeconomic benefits of using omega-3 fatty-acidenriched lipid emulsions have been investigated in Western populations, ${ }^{22}$ it is important to judge their effect in China.

The aim of this study was to perform a pharmacoeconomic evaluation of SMOFlipid (henceforth referred to as omega-3 fatty-acid-enriched lipid emulsion) vs standard parenteral nutrition within the Chinese ICU setting. To do this, we used a discrete event simulation model based on updated efficacy data from a previous international meta-analysis ${ }^{23}$ and Chinaspecific clinical and economic input parameters. ${ }^{24,25}$

\section{Materials and methods}

\section{Model structure}

The model was based on the discrete event simulation technique $^{26}$ and developed in Microsoft Excel (Microsoft Corp.,
Washington, USA). In a discrete event simulation, the experience of individuals is modeled over time in terms of the events that occur and the consequences of those events. Two alternative treatment arms were simulated: parenteral nutrition including 1) an omega-3 fatty-acid-enriched lipid emulsion (SMOFlipid, Fresenius Kabi, Bad Homburg, Germany) and 2) standard lipid emulsions. (Note: standard lipid emulsions were defined as lipid emulsions that were not enriched in omega-3 fatty-acids, such as soybean oil, medium-chain triglycerides/long-chain triglycerides, or olive oil/soybean oil emulsions.) Each simulated ICU patient was sent to both the omega-3 fatty-acid-enriched lipid emulsion and the standard lipid emulsion arms concurrently, so that the simulation of the two alternatives ran on the same patient cohort. Simulated patients entered the model at ICU admission, either directly or via the ward. Pre-ICU time was not differentiated between the two arms. The following events were considered: transfers from the ICU to the ward, new nosocomial infection, discharge from the hospital, and death, with discharge and death forming the two alternative ends to the patient pathway. Each iteration within the current study represents a unique patient and results were produced by calculating the statistics based on 10000 such simulated patients progressing through the simulation. Transition probabilities between these states were taken from the study of ICU patients in mainland China. ${ }^{24} \mathrm{~A}$ simplified model structure is shown in Figure 1. No discount rate was applied to outcomes and costs owing to the short time frame of the simulation.

\section{Patient population data sources}

We performed a targeted literature search for observational studies reporting clinical and/or economic outcomes for Chinese ICU patients, and reviewed the results, selecting studies that gave recent and comprehensive information on

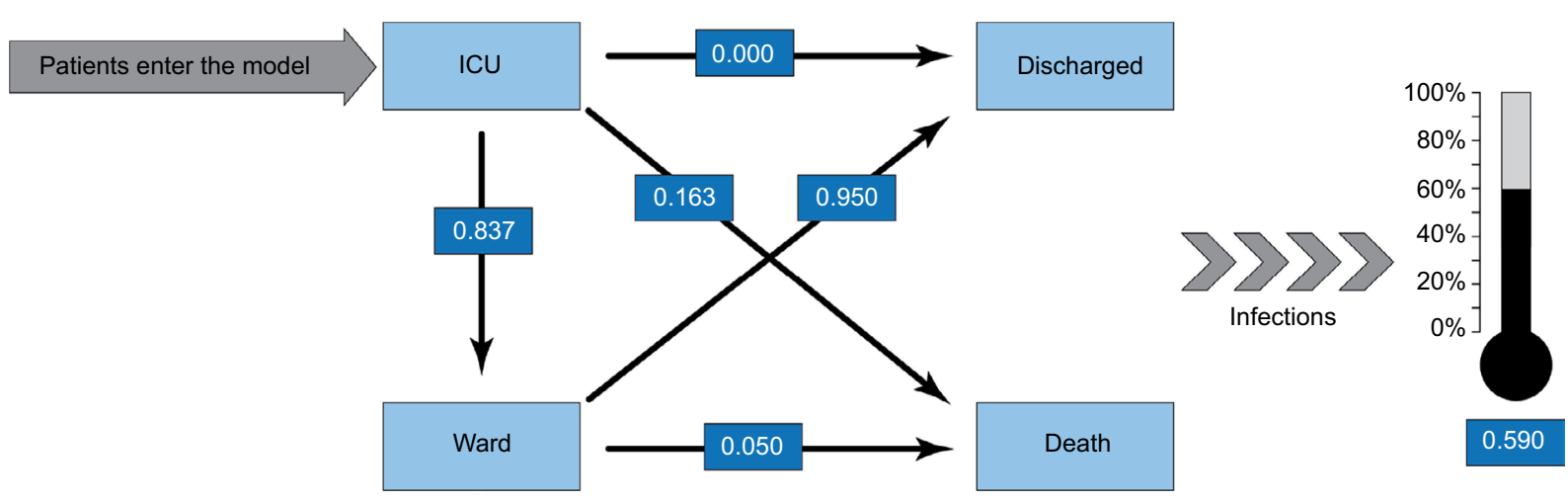

Figure I Model structure of transition probabilities.

Notes: Data from Du et al. ${ }^{24}$

Abbreviation: ICU, intensive care unit. 
the populations included in the model. Outcome data from $\mathrm{Du}$ et al were used for both the omega-3 fatty-acid-enriched and standard lipid emulsion patient populations included in the model. ${ }^{24}$ These data were converted into mean values according to the methods of Wan et al, ${ }^{27}$ and used for the standard lipid emulsion patient population as shown in Table 1. This table shows the probabilities of ICU patients' outcomes and mean length of stay, plus the expected mean length of parenteral nutrition. For the omega-3 fatty-acid-enriched lipid emulsion population, the same outcome data were partially recalculated to take into account the changes in clinical outcomes associated with this regimen, based on the meta-analysis conducted by Pradelli et $\mathrm{al}^{23}$ and its update (see section on Clinical efficacy data sources). Cost data were extracted from a recent publication reporting the daily cost of ICU and ward stay in Chinese hospitals (see section on Cost assessment data sources). ${ }^{25}$

Table I Probabilities of ICU patients' outcomes and mean lengths of stay for patients receiving standard parenteral nutrition.

\begin{tabular}{ll}
\hline Description & Value \\
\hline Probability of being transferred from ICU to a general & $83.7 \%$ \\
ward & \\
Probability of dying in the ICU & $16.3 \%$ \\
Probability of discharge from general ward & $95 \%$ \\
Probability of dying in the general ward & $5.0 \%$ \\
Probability of new infection acquired in the ICU & $59.0 \%$ \\
LOS, pre-ICU, days (SD) & $3.33(6.86)$ \\
LOS ICU - alive patients, days (SD) & $5.66(4.45)$ \\
LOS ICU - dead patients, days (SD) & $8.3(8.98)$ \\
LOS, post-ICU - alive patients, days (SD) & $13.32(13.1$ I) \\
LOS, post-ICU - dead patients, days (SD) & $32.67(34.10)$ \\
Duration of PN, days (SD) & $8.3(I 0.0)$ \\
\hline
\end{tabular}

Notes: Patients outcome and lengths of stay data from Du et al. ${ }^{24}$ Mean duration of parenteral nutrition was based on data from Wu et al. ${ }^{34}$

Abbreviations: ICU, intensive care unit; LOS, length of stay; PN, parenteral nutrition; SD, standard deviation.

\section{Clinical efficacy data sources}

The meta-analysis of Pradelli et $\mathrm{al}^{23}$ was updated in order to incorporate recent evidence into the evaluation. In brief, this update consisted of a systematic PubMed search using the same search criteria specified in Pradelli et al, ${ }^{23}$ but over a time period between August 2011 and November 2016. This procedure identified an additional five randomized controlled trials ${ }^{28-32}$ that contained clinical outcomes data on length of hospital or ICU stay or infections for ICU patients and which met eligibility criteria specified previously. ${ }^{23} \mathrm{New}$ meta-analyses were performed by pooling data using Review Manager (RevMan version 5.3; Copenhagen: The Nordic Cochrane Centre, The Cochrane Collaboration, 2014) from the studies identified by Pradelli et al, ${ }^{23}$ plus these five more recent studies, in a similar manner to the original study. ${ }^{23}$ The following results were obtained: infection risk reduction (RR) of 0.64 (95\% CI $0.47-0.87 ; p=0.004)$, reduction in ICU length of stay of 1.82 days $(95 \%$ CI $0.24-3.40 ; p=0.02)$, and reduction in post-ICU length of hospital stay of 3.07 days (95\% CI 1.01-5.13; $p=0.004$ ) (Figures 2-4, respectively). Absolute results for length of stay were converted into RR with standard deviations (SDs) as performed previously: ${ }^{22}$ 0.81 (SD 0.08) for ICU length of stay and 0.94 (SD 0.35) for post-ICU length of hospital stay. The effect of ICU mortality was taken into consideration when estimating a relative postICU length of stay, because not all ICU patients proceeded to the general ward.

\section{Cost assessment data sources}

The perspective of the study was that of the hospital. Only direct costs (e.g., cost of ICU or ward stay) rather than indirect costs were considered in the analysis. The time horizon corresponded to the hospital stay of ICU patients. Thus, the

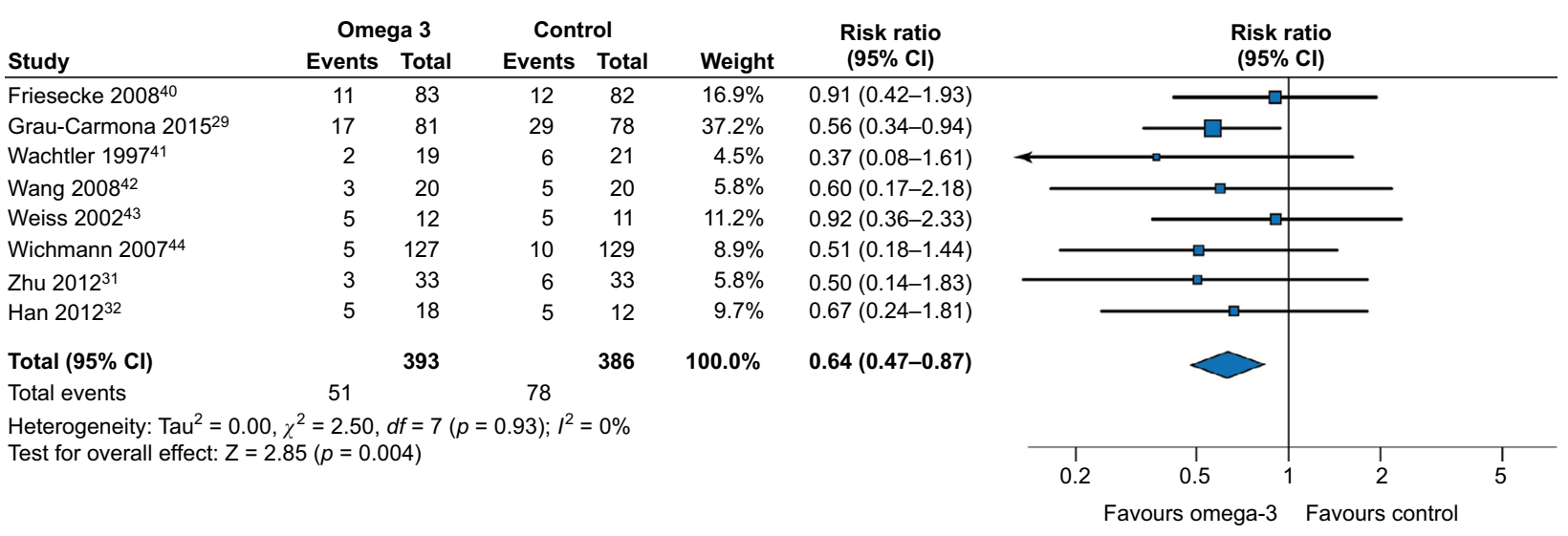

Figure 2 Infections: random effects meta-analysis and Forest plot for parenteral nutrition including omega-3 fatty-acid-enriched lipid emulsion vs standard parenteral nutrition not containing omega-3 fatty acids.

Notes: Squares represent individual study means of the effect measure; the pooled estimate is represented by a diamond symbol. 


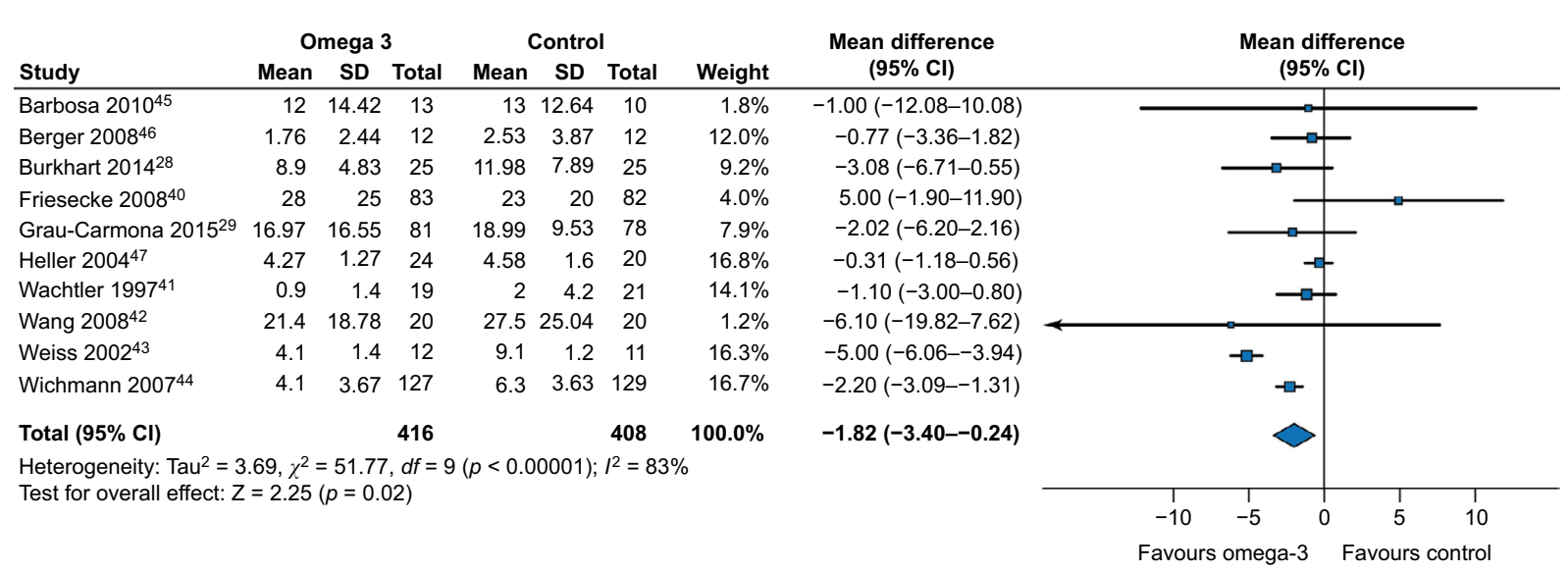

Figure 3 Length of intensive care unit stay: random effects meta-analysis and Forest plot for parenteral nutrition including omega-3 fatty-acid-enriched lipid emulsion vs standard parenteral nutrition not containing omega-3 fatty acids.

Notes: Squares represent individual study means of the effect measure; the pooled estimate is represented by a diamond symbol.

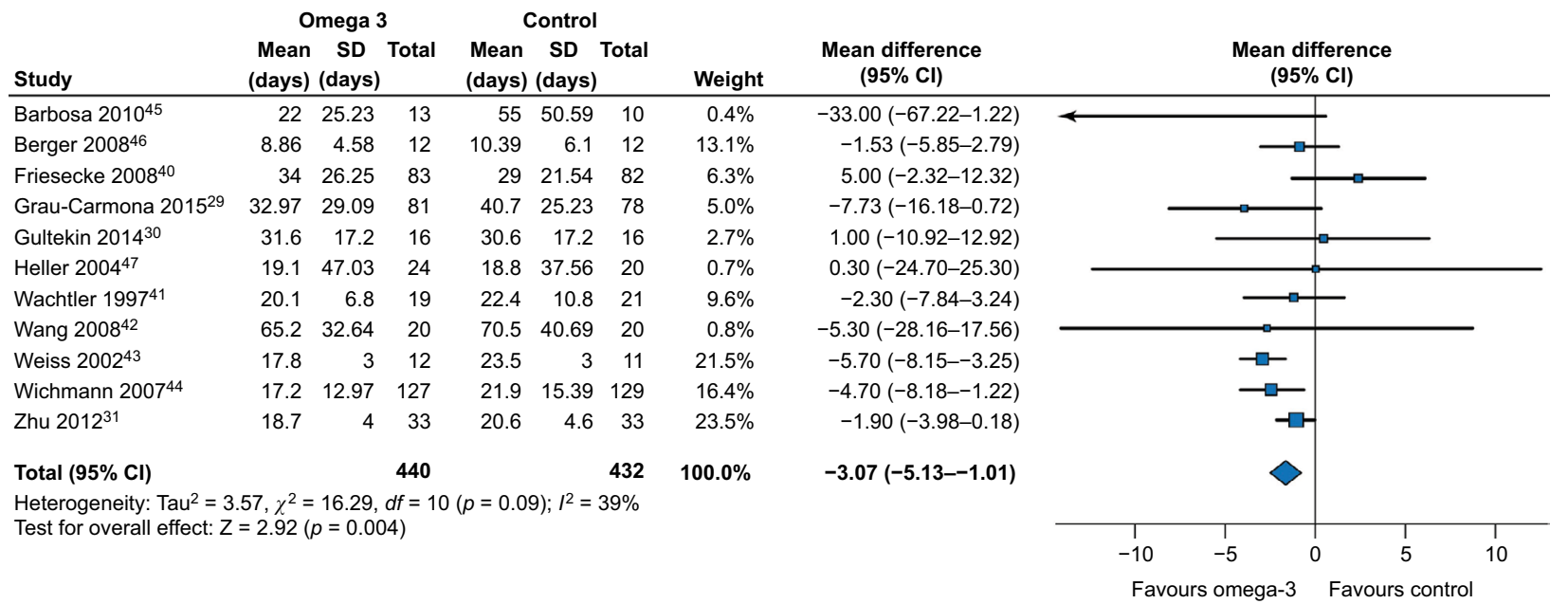

Figure 4 Post-intensive care unit length of hospital stay: random effects meta-analysis and Forest plot for parenteral nutrition including omega-3 fatty-acid-enriched lipid emulsion vs standard parenteral nutrition not containing omega- 3 fatty acids.

Notes: Squares represent individual study means of the effect measure; the pooled estimate is represented by a diamond symbol.

model included the following costs: 1) cost of omega-3 fattyacid-enriched lipid emulsions vs the cost of standard lipid emulsions not enriched with omega-3 fatty acids, 2) daily cost of ICU stay, 3) daily cost of general ward stay, and 4) cost of new infections acquired in ICU. All costs were presented in Chinese yuan renminbi (¥). Summaries of final financial results were also shown in US dollars using an exchange rate of $¥ 1$ to US $\$ 0.147$ for 10 July 2017.

Parenteral nutrition costs were calculated for every simulated patient on the basis of daily cost, which depended on the assigned treatment and duration of parenteral nutrition. Therefore, hospital prices of parenteral nutrition were weighted according to their national market share. ICU and ward-stay costs were taken from Tan et al, ${ }^{25}$ who reported daily hospital stay costs based on hospital financial reports for four main regions in China (Guangzhou, Beijing, Nanjing, and Xi'an). The average costs reported in 2013 across the four regions were actualized to current values by applying the official Chinese health care services inflation rate (2014, $1.5 \% ; 2015,1.2 \% ; 2016,3 \%) .{ }^{33}$ Thus, daily costs for the ICU and general ward used in the model were $¥ 6731.50$ (SD 1225.68 ) and $¥ 884.00$ (SD 103.51), respectively.

The cost of new infections was included as part of the model cost input, based on the extra antibiotic cost per nosocomial infection reported by $\mathrm{Wu}$ et $\mathrm{a}^{34}$ and actualized to 2016 figures by applying the $3.5 \%$ medication inflation rate for China during this period ( $¥ 4087$ ). ${ }^{33}$ These costs were limited to additional anti-infective treatments needed and did not include those associated with the effect of new infection on length of hospital stay or mortality rate; this aimed to avoid double counting, as length of stay and mortality rate were already included as clinical outcomes in the model. 


\section{Simulation and sensitivity analyses}

Sensitivity analyses were performed in order to validate the model's reliability. These involved changing one or more of the model parameters to see what effect this had on outcomes, thus assessing the sensitivity of the model to such changes. We performed a probabilistic sensitivity analysis and a oneway deterministic sensitivity analysis. The probabilistic sensitivity analysis showed the stability of the model outputs to several simultaneous changes in the parameters as it involved drawing all parameter values simultaneously from their probability distributions to create 1000 sets of unique parameter combinations (Monte Carlo simulations). SDs of $20 \%$ of mean values were used if no reliable uncertainty data were available. In the one-way deterministic sensitivity analysis a $\pm 20 \%$ variation was applied to model parameter values one by one, keeping the other values constant, to see how sensitive the model results were to relatively extreme changes.

\section{Results}

Base-case model outcomes (hospital length of stay and infection rate) for 10000 simulated patients showed that parenteral nutrition enriched with omega-3 fatty-acid lipid emulsions had the following efficacy advantages over parenteral nutrition with standard (non-omega-3 fatty-acid enriched) lipid emulsions: 1) prevention of more than one-third (2109 of 5932; 35.6\%) of infections in ICU patients and 2) reduction of ICU stay by 1.15 days and ward stay by 0.71 days, resulting in a reduction of overall length of hospital stay by 1.87 days for ICU patients (Table 2). Thus, omega-3 fatty-acid-enriched lipid emulsions

Table 2 Mean clinical outcome parameter results from model simulations

\begin{tabular}{|c|c|c|c|}
\hline Parameter & $\mathbf{S T}+\mathbf{\Omega 3}$ & ST & Difference \\
\hline LOS - total, days (SD) & $19.48(16.46)$ & $21.35(17.52)$ & $-1.87(1.38)$ \\
\hline Ward (pre-ICU) & $3.31(7.00)$ & $3.31(7.00)$ & 0 \\
\hline ICU & $5.03(4.43)$ & $6.18(5.50)$ & $-1.15(1.07)$ \\
\hline Ward (post-ICU) & $11.14(14.48)$ & $11.85(15.41)$ & $-0.71(0.93)$ \\
\hline $\begin{array}{l}\text { Infections per } 10000 \\
\text { patients (SD) }\end{array}$ & $3822(4859)$ & $5931(4913)$ & $-2109(4080)$ \\
\hline
\end{tabular}

Abbreviations: ICU, intensive care unit; LOS, length of stay; SD, standard deviation; ST, standard parenteral nutrition; $\mathrm{ST}+\Omega 3$, fish-oil-enriched parenteral nutrition.

Table 3 Results of the cost-effectiveness analysis: mean costs per patient (standard deviation)

\begin{tabular}{|c|c|c|c|}
\hline Parameter & $\mathbf{S T}+\mathbf{\Omega 3}$ & ST & Difference \\
\hline Total cost, $¥$ & 47 I89 (30 950) & $54783(37$ I83) & $-7594(7005)$ \\
\hline ICU cost, $¥$ & 32001 (28 228) & 39349 (34 997) & $-7348(6779)$ \\
\hline Ward (pre-ICU) cost, $¥$ & $277 \mid(5885)$ & 277 I (5885) & $0(0)$ \\
\hline Ward cost, $¥$ & 9306 (12 099) & $9897(12874)$ & $-59 \mid(775)$ \\
\hline Infection cost, $¥$ & $1562(1986)$ & $2424(2008)$ & $-862(1668)$ \\
\hline Treatment cost, $¥$ & 1549 (1339) & $342(294)$ & $1207(1047)$ \\
\hline ICER, ¥/LOS day & & & Dominant \\
\hline
\end{tabular}

Abbreviations: ICER, incremental cost-effectiveness ratio (ST + $\Omega 3$ vs ST); ICU, intensive care unit; LOS, length of stay; ST, standard parenteral nutrition; ST + $\Omega 3$, fishoil-enriched parenteral nutrition.

ClinicoEconomics and Outcomes Research 2017:9 


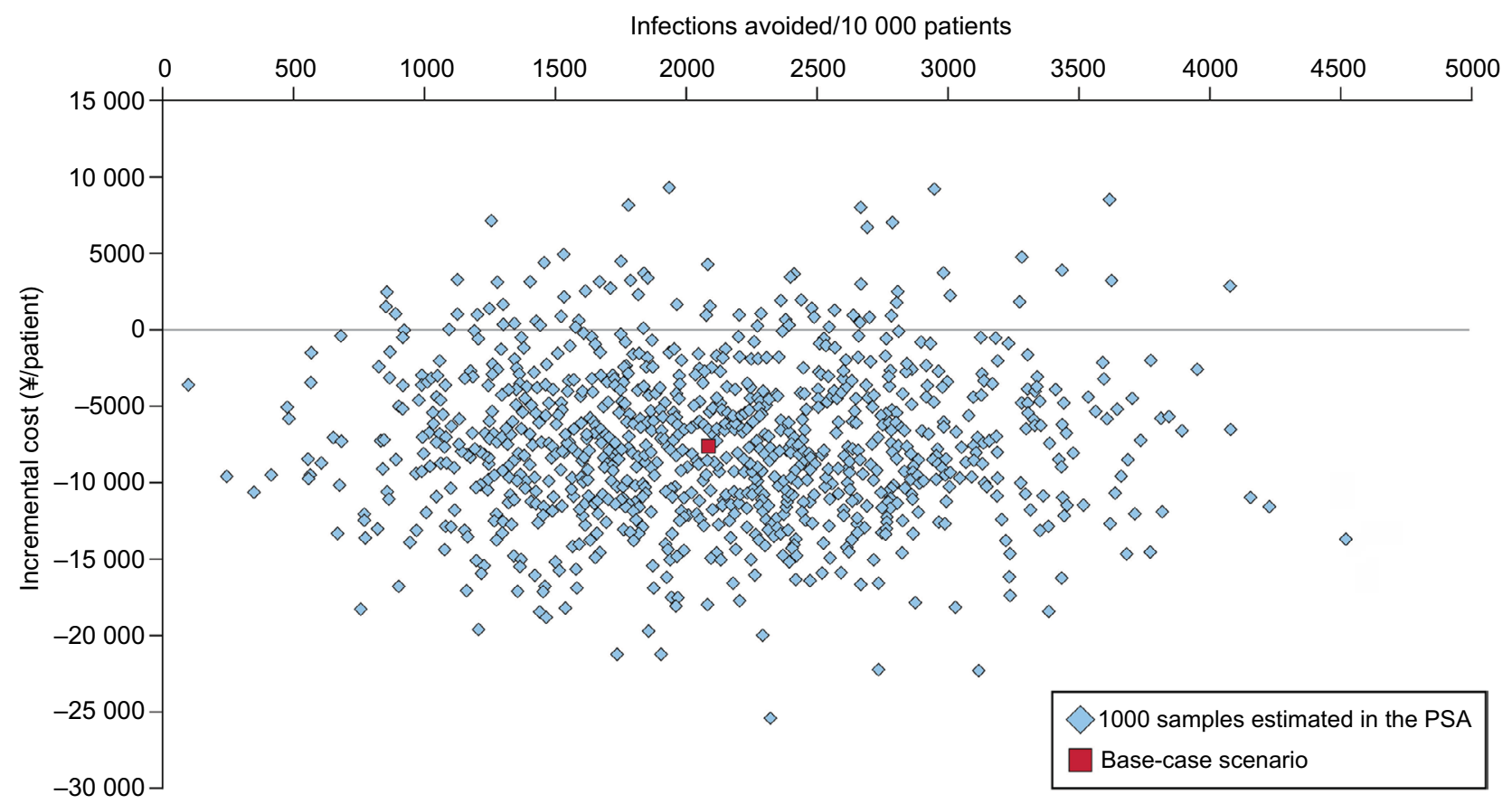

Figure 5 Scatterplot of the results of the probabilistic sensitivity analysis for parenteral nutrition including omega-3 fatty-acid-enriched lipid emulsions vs standard parenteral nutrition not containing omega-3 fatty acids.

Abbreviation: PSA, probabilistic sensitivity analysis.

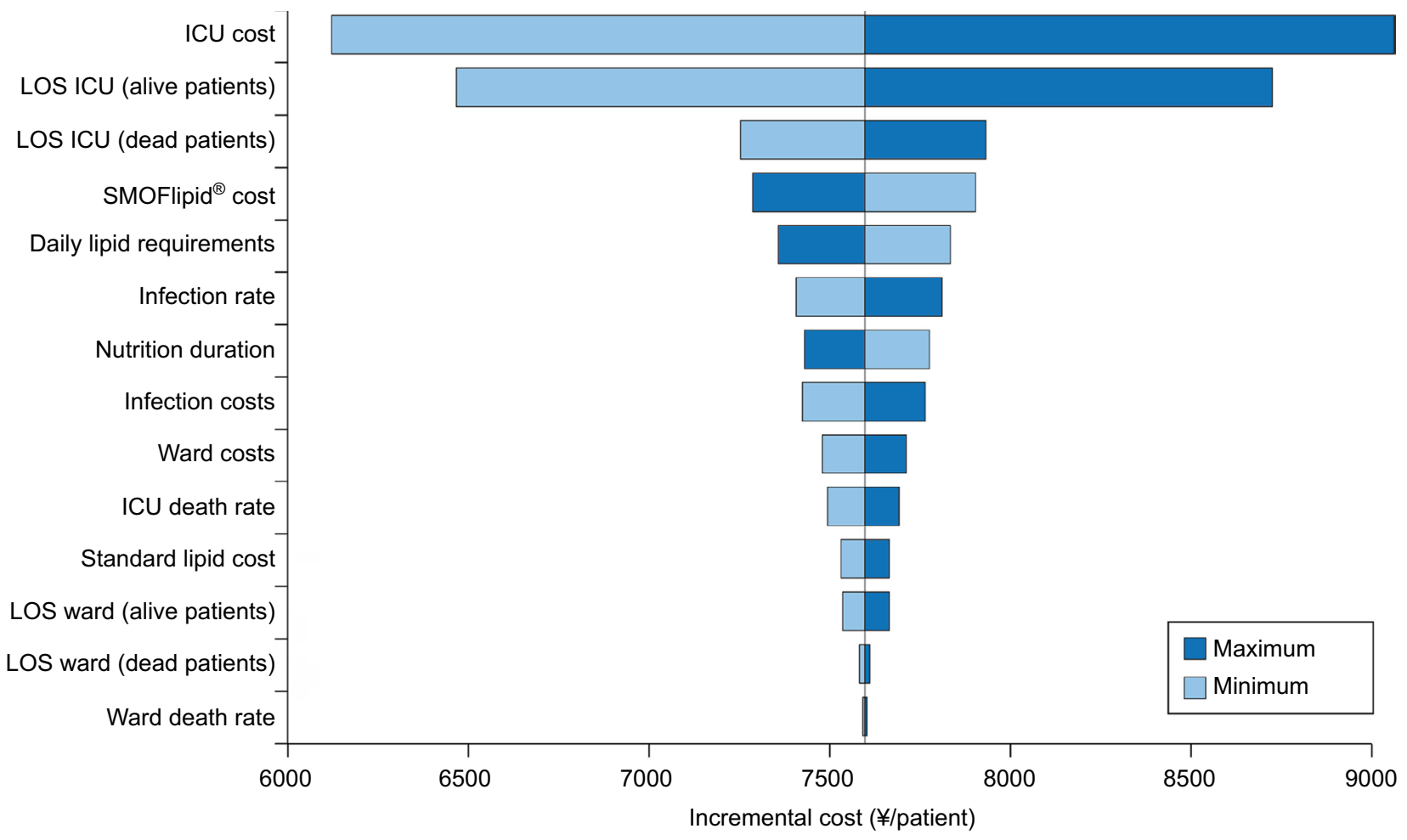

Figure 6 Tornado plot of deterministic sensitivity analysis results on the difference in total cost ( $¥)$ between standard parenteral nutrition not containing omega-3 fatty acids minus parenteral nutrition with omega-3 fatty-acid-enriched lipid emulsions.

Abbreviations: ICU, intensive care unit; LOS, length of stay. 
on average, omega-3 fatty-acid-enriched parenteral nutrition is a cost-saving strategy in the Chinese ICU setting.

\section{Discussion}

We conducted a cost-effectiveness analysis using clinical outcomes from an update to our previous meta-analysis, together with financial data from Chinese hospitals. Our costeffectiveness analysis results showed that parenteral nutrition including an omega-3 fatty-acid-enriched lipid emulsion could significantly improve clinical outcomes in ICU patients in China, and also lead to cost savings, compared with standard parenteral nutrition. This was because the additional acquisition cost of omega-3 fatty-acid-enriched lipid emulsions was completely offset by reduced costs associated with shorter ICU and hospital stays and fewer hospital-emergent infections. In fact, the model results predicted a mean cost saving of $¥ 7594$ (US \$1116) per patient given parenteral nutrition including omega-3 fatty-acid-enriched lipid emulsions compared with standard parenteral nutrition regimens.

We have confidence in these results for a number of reasons. First, the observed clinical benefits of using lipid emulsions enriched with omega-3 fatty acids are in accordance with other similar meta-analyses. Here, we reported reductions of more than one-third $(35.6 \%)$ for infection rates and 1.87 days' reduction in overall length of hospital stay for ICU patients. In Pradelli et al, ICU infections were reduced by $29 \%$ and length of hospital stay by 5.17 days for ICU patients. ${ }^{23}$ Other meta-analyses for ICU populations have shown significant reductions in overall length of hospital stay ${ }^{35,36}$ and in infectious complications ${ }^{35}$ when using parenteral nutrition including lipid emulsions enriched with omega-3 fatty acids compared with standard parenteral nutrition. In the current study, the sensitivity analyses demonstrated that the model results were robust. The probabilistic sensitivity analysis results (Figure 5) showed that the group receiving omega-3 fatty-acid-enriched lipid emulsions was less costly than the control group in $93 \%$ of the simulations. Moreover, none of the deterministic sensitivity analysis variations altered the conclusion that parenteral nutrition with omega-3 fatty-acid-enriched lipid emulsions was a cost-saving strategy in the Chinese ICU setting (Figure 6). Finally, the cost-effectiveness analysis model used in the current study has been used previously for related studies. A previous pharmacoeconomic study within the Chinese health care setting showed that the addition of omega-3 fatty-acid-enriched lipid emulsion (Omegaven ${ }^{\circledR}$ 10\% fish oil emulsion; Fresenius Kabi, Bad Homburg, Germany) to standard parenteral nutrition improved clinical outcome and decreased overall costs in Chinese ICU patients. ${ }^{34}$ Moreover, a similar pharmacoeconomic study demonstrated that parenteral nutrition regimens with omega-3 fatty acids would be cost effective in Italian, French, German, and UK hospitals for ICU and non-ICU patients. ${ }^{22}$

Limitations of the present study are generally those that are inherent to a modeling approach, such as a need to combine different data sources within one logical construct. However, modeling use in economic evaluations is recognized as an unavoidable fact of life. ${ }^{37}$ This is because models can represent the complexities of everyday life in a more simple and understandable manner, and are thus often used in health economic evaluations where clinical trials are missing or if they did not include economic data. In these cases decision analytic models are used to synthesize the best available data. ${ }^{37}$ We used a decision model, as this type of model is useful for combining or linking data from different research areas and sources and/or transferring or extrapolating results from one time, place, population, or setting, to another. ${ }^{38}$ Another potential weakness is that the relative effectiveness estimates applied to our patient population were derived from international studies conducted on a mixed, international ICU patient population. However, the applicability of similar data to Chinese standards of care was validated externally in our previous study using observations from the Chinese setting, increasing the credibility of our modeling approach and data sources. ${ }^{34}$ Finally, models must be based on high-quality evidence in order to produce accurate and reliable results. In the current model, we employed the standard approach of utilizing meta-analysis data to increase sample size and thus the power to detect effects of interest. To minimize bias all studies included in the meta-analysis were rated for methodological quality using the modified Jadad scale. ${ }^{39}$ The studies used to provide clinical data for this cost-effectiveness analysis were all rated as good to excellent quality (Jadad scale $\geq 3$ ).

In conclusion, we have described a pharmacoeconomic evaluation of an omega-3 fatty-acid-enriched lipid emulsion vs standard parenteral nutrition within the Chinese ICU setting. The model results indicate that, within this setting, an omega-3 fatty-acid-enriched lipid emulsion has the potential to significantly improve clinical outcomes and also reduce health care costs. This is because the modest additional acquisition costs of omega-3 fatty-acid-enriched lipid emulsions are more than offset by savings in a reduced length of hospital stay and, to a lesser extent, by reductions in the likelihood of nosocomial infection. The model shows that omega-3 fattyacid-enriched lipid emulsions provide a better quality of patient care for less cost in $93 \%$ of (simulated) ICU patients, 
so parenteral nutrition with omega-3 fatty-acid-enriched lipid emulsions is said to "dominate" standard parenteral nutrition. Thus, omega-3 fatty-acid-enriched lipid emulsions create a win-win scenario for both health care providers and patients, and should thus become standard care under the circumstances and conditions covered by this cost-effectiveness model.

\section{Acknowledgments}

The authors thank Dr Richard Clark (freelance medical writer, Dunchurch, Warwickshire, UK) for writing this manuscript, and Dr Martina Sintzel (mcs medical communication services, Kusnacht, Switzerland) for valuable consultation services.

\section{Disclosure}

Dr Lorenzo Pradelli is a director and an employee of AdRes, which has received project funding from Fresenius Kabi. The authors report no conflicts of interest in this work.

\section{References}

1. Norman K, Pichard C, Lochs H, Pirlich M. Prognostic impact of diseaserelated malnutrition. Clin Nutr. 2008;27:5-15.

2. Pirlich M, Schutz T, Norman K, et al. The German hospital malnutrition study. Clin Nutr. 2006;25(4):563-572.

3. Sorensen J, Kondrup J, Prokopowicz J, et al. EuroOOPS: an international, multicentre study to implement nutritional risk screening and evaluate clinical outcome. Clin Nutr. 2008;27(3):340-349.

4. Schneider SM, Veyres P, Pivot X, et al. Malnutrition is an independent factor associated with nosocomial infections. Br J Nutr. 2004;92(1): $105-111$.

5. Fry DE, Pine M, Jones BL, Meimban RJ. Patient characteristics and the occurrence of never events. Arch Surg. 2010;145(2):148-151.

6. Barker LA, Gout BS, Crowe TC. Hospital malnutrition: prevalence, identification and impact on patients and the healthcare system. Int $J$ Environ Res Public Health. 2011;8(2):514-527.

7. Fang S, Long J, Tan R, et al. A multicentre assessment of malnutrition, nutritional risk, and application of nutritional support among hospitalized patients in Guangzhou hospitals. Asia Pac J Clin Nutr. 2013;22(1):54-59.

8. Wu BW, Yin T, Cao WX, et al. Clinical application of subjective global assessment in Chinese patients with gastrointestinal cancer. World $J$ Gastroenterol. 2009;15(28):3542-549.

9. Zhang L, Wang X, Huang Y, et al. NutritionDay 2010 audit in Jinling hospital of China. Asia Pac J Clin Nutr. 2013;22(2):206-213.

10. Wu GH, Liu ZH, Zheng LW, Quan YJ, Wu ZH. Prevalence of malnutrition in general surgical patients: evaluation of nutritional status and prognosis. Zhonghua Wai Ke Za Zhi. 2005;43(11):693-696.

11. Thibault R, Heidegger CP, Berger MM, Pichard C. Parenteral nutrition in the intensive care unit: cautious use improves outcome. Swiss Med Wkly. 2014;144:w13997.

12. Villet S, Chiolero RL, Bollmann MD, et al. Negative impact of hypocaloric feeding and energy balance on clinical outcome in ICU patients. Clin Nutr. 2005;24(4):502-509.

13. Jie B, Jiang ZM, Nolan MT, et al. Impact of nutritional support on clinical outcome in patients at nutritional risk: a multicenter, prospective cohort study in Baltimore and Beijing teaching hospitals. Nutrition. 2010;26(11-12):1088-1093.

14. Kruizenga HM, van Tulder MW, Seidell JC, Thijs A, Ader HJ, van Bokhorst-de van der Schueren MA. Effectiveness and cost-effectiveness of early screening and treatment of malnourished patients. Am J Clin Nutr. 2005;82(5):1082-1089.
15. Martin CM, Doig GS, Heyland DK, Morrison T, Sibbald WJ; Southwestern Ontario Critical Care Research Network. Multicentre, clusterrandomized clinical trial of algorithms for critical-care enteral and parenteral therapy (ACCEPT). CMAJ. 2004;170(2):197-204.

16. Elia M, Evans E, Russell C, et al. The cost of malnutrition in England and the potential cost savings from nutritional interventions (short version). Redditch, UK: BAPEN, 2015. Available from: http://www. bapen.org.uk. Accessed April 15, 2017.

17. Singer P, Berger MM, Van den Berghe G, et al. ESPEN guidelines on parenteral nutrition: intensive care. Clin Nutr. 2009;28(4):387-400.

18. Braga M, Ljungqvist O, Soeters $\mathrm{P}$, Fearon K, Weimann A, Bozzetti F. ESPEN guidelines on parenteral nutrition: surgery. Clin Nutr. 2009;28(4):378-386.

19. Schade I, Rohm KD, Schellhaass A, Mengistu A, Boldt J, Piper SN. Inflammatory response in patients requiring parenteral nutrition: comparison of a new fish-oil-containing emulsion $\left(\mathrm{SMOF}^{\circledR}\right)$ versus an olive/ soybean oil-based formula. P144. Crit Care. 2008;12(Suppl 2):S56-S57.

20. Lin HG, Li N, Wang XY, Zhu WM, Jiang J, Li JS. Effect of SMOF fat emulsions on fatty acid profile and inflammatory mediator of in major abdominal surgical patients. Parenter Enteral Nutr. 2010;17:195-198.

21. Metry AA, Abdelaal W, Ragaei M, Refaat M, Nakhla G. Smoflipid versus intralipid in postoperative ICU patients. Enliven: JAnaesthesiol Crit Care Med. 2014;1(6):015.

22. Pradelli L, Eandi M, Povero M, et al. Cost-effectiveness of omega-3 fatty acid supplements in parenteral nutrition therapy in hospitals: a discrete event simulation model. Clin Nutr. 2014;33(5):785-792.

23. Pradelli L, Mayer K, Muscaritoli M, Heller AR. n-3 fatty acid-enriched parenteral nutrition regimens in elective surgical and ICU patients: a meta-analysis. Crit Care. 2012;16(5):R184.

24. Du B, An Y, Kang Y, et al. Characteristics of critically ill patients in ICUs in mainland China. Crit Care Med. 2013;41:84-92.

25. Tan SC, Wang X, Wu B, et al. Cost-effectiveness of linezolid versus vancomycin among patients with methicillin-resistant Staphylococcus aureus confirmed nosocomial pneumonia in China. Value Health Reg Issues. 2014;3:94-100.

26. Caro JJ. Pharmacoeconomic analyses using discrete event simulation. Pharmacoeconomics. 2005;23:323-332.

27. Wan X, Wang W, Liu J, Tong T. Estimating the sample mean and standard deviation from the sample size, median, range and/or interquartile range. BMC Med Res Methodol. 2014;14:135.

28. Burkhart CS, Dell-Kuster S, Siegemund M, et al. Effect of n-3 fatty acids on markers of brain injury and incidence of sepsis-associated delirium in septic patients. Acta Anaesthesiol Scand. 2014;58: 689-700.

29. Grau-Carmona T, Bonet-Saris A, Garcia-de-Lorenzo A, et al. Influence of n-3 polyunsaturated fatty acids enriched lipid emulsions on nosocomial infections and clinical outcomes in critically ill patients: ICU lipids study. Crit Care Med. 2015;43:31-39.

30. Gultekin G, Sahin H, Inanc N, Uyanik F, Ok E. Impact of omega-3 and omega- 9 fatty acids enriched total parenteral nutrition on blood chemistry and inflammatory markers in septic patients. PakJ Med Sci. 2014;30:299-304.

31. Zhu, XH, Wu YF, Qiu YD, Jiang CP, Ding YT. Liver-protecting effects of omega-3 fish oil lipid emulsion in liver transplantation. World $J$ Gastroenterol. 2012:18;6141-6147.

32. Han YY, Lai SL, Ko WJ, Chou CH, Lai HS. Effects of fish oil on inflammatory modulation in surgical intensive care unit patients. Nutr Clin Pract. 2012;27:91-98.

33. National Bureau of Statistics of China. Available from: http://www. stats.gov.cn/english/. Accessed April 15, 2017.

34. Wu GH, Gao J, Ji CY, Pradelli L, Xi QL, Zhuang QL. Cost and effectiveness of omega-3 fatty acid supplementation in Chinese ICU patients receiving parenteral nutrition. Clinicoecon Outcomes Res. 2015;7:369-375.

35. Manzanares W, Langlois PL, Dhaliwal R, Lemieux M, Heyland DK. Intravenous fish oil lipid emulsions in critically ill patients: an updated systematic review and meta-analysis. Crit Care. 2015;19:167. 
36. Palmer AJ, Ho CKM, Ajibola O, Avenell A. The role of omega-3 fatty acid supplemented parenteral nutrition in critical illness in adults: a systematic review and meta-analysis. Crit Care Med. 2013;41 307-316.

37. Buxton MJ, Drummond MF, Van Hout BA, et al. Modelling in economic evaluation: an unavoidable fact of life. Health Econ. 1997;6: 217-227.

38. Siebert U. When should decision-analytic modeling be used in the economic evaluation of health care? Eur J Health Econ. 2003;4: $143-150$.

39. Oremus M, Wolfson C, Perrault A, Demers L, Momoli F, Moride Y. Interrater reliability of the modified Jadad quality scale for systematic reviews of Alzheimer's disease drug trials. Dement Geriatr Cogn Disord. 2001;12:232-236.

40. Friesecke S, Lotze C, Köhler J, Heinrich A, Felix SB, Abel P. Fish oil supplementation in the parenteral nutrition of critically ill medical patients: a randomised controlled trial. Intensive Care Med. 2008;34(8):1411-1420.

41. Wachtler P, König W, Senkal M, Kemen M, Köller M. Influence of a total parenteral nutrition enriched with omega-3 fatty acids on leukotriene synthesis of peripheral leukocytes and systemic cytokine levels in patients with major surgery. J Trauma. 1997;42(2):191-198.
42. Wang X, Li W, Li N, Li J. Omega-3 fatty acids-supplemented parenteral nutrition decreases hyperinflammatory response and attenuates systemic disease sequelae in severe acute pancreatitis: a randomized and controlled study. JPEN J Parenter Enteral Nutr. 2008;32(3):236-241.

43. Weiss G, Meyer F, Matthies B, Pross M, Koenig W, Lippert H. Immunomodulation by perioperative administration of $\mathrm{n}-3$ fatty acids. $\mathrm{Br} J$ Nutr. 2002;87:S89-94.

44. Wichmann MW, Thul P, Czarnetzki HD, Morlion BJ, Kemen M, Jauch KW. Evaluation of clinical safety and beneficial effects of a fish oil containing lipid emulsion (Lipoplus, MLF541): data from a prospective, randomized, multicenter trial. Crit Care Med. 2007;35(3):700-706.

45. Barbosa VM, Miles EA, Calhau C, Lafuente E, Calder PC. Effects of a fish oil containing lipid emulsion on plasma phospholipid fatty acids, inflammatory markers, and clinical outcomes in septic patients: a randomized, controlled clinical trial. Crit Care. 2010;14(1):R5.

46. Berger MM, Tappy L, Revelly JP, Koletzko BV, Gepert J, Corpataux JM, Cayeux MC, Chiolero RL. Fish oil after abdominal aorta aneurysm surgery. Eur J Clin Nutr. 2008;62(9):1116-1122.

47. Heller AR, Rössel T, Gottschlich B, Tiebel O, Menschikowski M, Litz RJ, Zimmermann T, Koch T. Omega-3 fatty acids improve liver and pancreas function in postoperative cancer patients. Int $J$ Cancer. 2004;111(4):611-616.
ClinicoEconomics and Outcomes Research

\section{Publish your work in this journal}

ClinicoEconomics and Outcomes Research is an international, peerreviewed open-access journal focusing on health technology assessment, pharmacoeconomics and outcomes research in the areas of diagnosis, medical devices, and clinical, surgical and pharmacological intervention. The economic impact of health policy and health systems
Submit your manuscript here: https://www.dovepress.com/clinicoeconomics-and-outcomes-research-journal

\section{Dovepress}

organization also constitute important areas of coverage. The manuscript management system is completely online and includes a very quick and fair peer-review system, which is all easy to use. Visit http://www.dovepress.com/testimonials.php to read real quotes from published authors. 